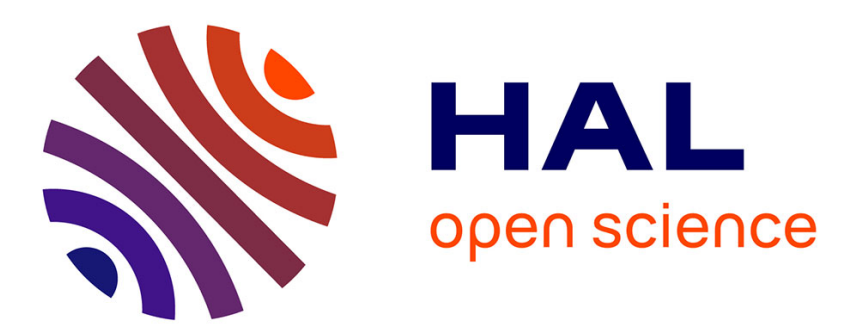

\title{
Dynamics of the localization of the plastid terminal oxidase PTOX inside the chloroplast
}

Susanne Bolte, Elodie Marcon, Mélanie Jaunario, Lucas Moyet, Marie-Thérèse Paternostre, Marcel Kuntz, Anja Krieger-Liszkay

\section{To cite this version:}

Susanne Bolte, Elodie Marcon, Mélanie Jaunario, Lucas Moyet, Marie-Thérèse Paternostre, et al.. Dynamics of the localization of the plastid terminal oxidase PTOX inside the chloroplast. Journal of Experimental Botany, 2020, 71 (9), pp.2661-2669. 10.1093/jxb/eraa074 . hal-02495692

\section{HAL Id: hal-02495692 \\ https://hal.science/hal-02495692}

Submitted on 20 Oct 2020

HAL is a multi-disciplinary open access archive for the deposit and dissemination of scientific research documents, whether they are published or not. The documents may come from teaching and research institutions in France or abroad, or from public or private research centers.
L'archive ouverte pluridisciplinaire HAL, est destinée au dépôt et à la diffusion de documents scientifiques de niveau recherche, publiés ou non, émanant des établissements d'enseignement et de recherche français ou étrangers, des laboratoires publics ou privés. 
2

3 Susanne Bolte ${ }^{1}$, Elodie Marcon $^{2}$, Mélanie Jaunario ${ }^{2}$, Lucas Moyet $^{3}$, Marie-Thérèse 4 Paternostre ${ }^{2}$, Marcel Kuntz ${ }^{3}$, Anja Krieger-Liszkay ${ }^{2 * *}$

5

6

\section{Facility, Paris, France}

8 2Université Paris-Saclay, CEA, CNRS, Institute for Integrative Biology of the Cell (I2BC),

9 91198, Gif-sur-Yvette cedex, France

10

\section{Dynamics of the localization of the plastid terminal oxidase PTOX inside the chloroplast}

${ }^{1}$ Sorbonne Université, CNRS-FRE 3631 - Institut de Biologie Paris Seine, Imaging Core ${ }^{3}$ Cell \& Plant Physiology Laboratory, Université Grenoble Alpes, CNRS, INRA, CEA, 17-

Rue des Martyrs, 38054 Grenoble cedex 9, France

2

*Author for correspondence: Anja Krieger-Liszkay; e-mail anja.krieger-liszkay@cea.fr

Running title: Localization of the plastid terminal oxidase

\section{High light}

We demonstrate that plastid terminal oxidase (PTOX) localization to the thylakoid membrane depends on $\mathrm{pH}$ and ionic strength using in vitro reconstitution assays and GFP-labelled PTOX in confocal microscopy.

Word count: 4433 
The plastid terminal oxidase (PTOX) is a plastohydroquinone:oxygen oxidoreductase that shares structural similarities with alternative oxidases (AOX). Multiple roles have been attributed to PTOX, such as involvement in carotene desaturation, a safety valve function, participation in the processes of chlororespiration and setting the redox poise for cyclic electron transport. PTOX activity has been previously shown to depend on its localization at the thylakoid membrane. Here we investigated the dynamics of PTOX localization in dependence on the proton motive force. Infiltrating illuminated leaves with uncouplers led to a partial dissociation of PTOX from the thylakoid membrane. In vitro reconstitution experiments showed that the attachment of purified recombinant MBP-OsPTOX to liposomes and isolated thylakoid membranes was strongest at slightly alkaline $\mathrm{pH}$ values in the presence of lower millimolar concentrations of $\mathrm{KCl}$ or $\mathrm{MgCl}_{2}$. In A. thaliana overexpressing GFP-PTOX, confocal microscopy images showed that PTOX formed distinct spots in chloroplasts of darkadapted or uncoupler-treated leaves while the protein was more equally distributed in a network-like structure in the light. We propose a dynamic PTOX association with the thylakoid membrane depending on the presence of a proton motive force.

Keywords: alternative electron transport; chloroplast; confocal microscopy; liposomes; membrane association; plastid terminal oxidase 


\section{Introduction}

The plastid terminal oxidase (PTOX) is a non-heme diiron quinol oxidase that oxidizes plastoquinol and reduces $\mathrm{O}_{2}$ to $\mathrm{H}_{2} \mathrm{O}$. PTOX was discovered in the so-called immutans mutant of A. thaliana showing a variegated phenotype (Wetzel et al., 1994; Carol et al., 1999). This enzyme is involved in carotenoid biosynthesis, plastid development, chlororespiration, and it has been proposed to act as a safety valve by protecting the plastoquinone pool from overreduction under abiotic stress. A. thaliana grown in moderate light under non-stress conditions have low PTOX concentrations (about 1 PTOX protein per 100 PSII; Lennon et al., 2003). By contrast, elevated PTOX levels have been found in certain species exposed to abiotic stresses such as high temperatures, high light and drought (Quiles, 2006), salinity (Stepien and Johnson, 2009; 2018), low temperatures, and high intensities of visible (Ivanov et al., 2012) and UV light (Laureau et al., 2013).

It is generally accepted that in most plant species PTOX has low activity compared to photosynthetic electron flow. The maximum rate of PTOX was reported to be $5 \mathrm{e}^{-} \mathrm{s}^{-1}$ per PSII for PTOX2 in C. reinhardtii and $0.3 \mathrm{e}^{-} \mathrm{s}^{-1} \mathrm{PSII}^{-1}$ in tomato (Trouillard et al., 2012), while the maximal rate of photosynthesis is approximately $150 \mathrm{e}^{-} \mathrm{s}^{-1}$ per PSII (Nawrocki et al., 2015). However, in Eutrema salsugineum exposed to stress, PTOX activity can account for $30 \%$ of the PSII activity (Stepien and Johnson, 2009). The in vitro enzyme activity of recombinant PTOX (MBP-OsPTOX) from rice is high (up to $19.01 \pm 1.1 \mu \mathrm{mol} \mathrm{O} \mathrm{O}_{2}$ protein ${ }^{-1} \mathrm{~min}^{-1}$; Yu et al., 2014). The discrepancy between the reported PTOX activities in planta and the $\mathrm{V}_{\max }$ measured with the purified protein points to a mechanism that allows the regulation of PTOX activity depending on the reduction state of the electron transport chain.

Since PTOX may compete with linear and cyclic electron flow (Feilke et al., 2016; Krieger-Liszkay and Feilke, 2016), a highly active or highly abundant PTOX may negatively impact ATP and NADPH production. To avoid interference of PTOX activity with photosynthetic electron transport under conditions favorable for $\mathrm{CO}_{2}$ assimilation, its activity must be tightly controlled. High PTOX activity seems to be beneficial for the plant to protect the photosynthetic apparatus against photoinhibition when the electron transport chain is in a highly reduced state, as it is the case under abiotic stress like high salinity, drought, or when $\mathrm{CO}_{2}$ fixation is limited by unfavorable temperatures. However, high PTOX activity is detrimental to high photosynthetic activity when light and $\mathrm{CO}_{2}$ are not limiting.

PTOX has been localized in spinach chloroplasts of non-stressed leaves in the nonappressed regions of the thylakoid membrane (Lennon et al., 2003). We have proposed previously a model suggesting that PTOX attachment to the thylakoid membrane depends on 
the proton motive force (Krieger-Liszkay and Feilke, 2016). According to this model, PTOX is attached to the membrane in high light when both $\Delta \mathrm{pH}$ (the proton gradient) and $\Delta \psi$ (the membrane potential) across the thylakoid membrane are high. Changes of the localization of PTOX would regulate PTOX activity by either allowing or by restricting its access to its substrate. We have shown previously that PTOX was associated to thylakoid membrane isolated from leaves exposed to high light, but not in those isolated from dark-adapted leaves. In dark-adapted leaves PTOX was mostly found in the fraction of soluble proteins (Feilke et al., 2016). Recently another type of regulation also based on changes in PTOX localization has been described in Eutrema salsugineum exposed to salt stress. In control conditions, PTOX was localized at the unstacked stroma lamellae where it has no access to its substrate plastoquinol while in plants exposed to salt stress it was translocated to the grana stacks (Stepien and Johnson, 2018). Stepien and Johnson (2018) proposed that PTOX not only translocates from the stroma lamellae to the grana stacks but may also moves across the thylakoid membrane to the lumen side and is active in the lumen.

Here we studied the effect of the proton motive force on PTOX attachment to the membrane by different approaches. We infiltrated leaves with uncouplers and determined the PTOX localization by immunoblots. In addition, we adsorbed recombinant MBP-OsPTOX to liposomes or thylakoid membranes at different $\mathrm{pH}$ values and different ion concentrations. Confocal microscopy images revealed differences in the localization of GFP-PTOX between leaves of dark- and light-adapted $A$. thaliana plants.

\section{Materials and Methods}

\section{Plant Material}

A. thaliana (Col-0) was grown for 6-8 weeks in soil in a $8 \mathrm{~h}$ light $\left(140 \mu \mathrm{mol}\right.$ photons $\left.\mathrm{m}^{-2} \mathrm{~s}^{-1}\right)$, $22^{\circ} \mathrm{C} / 16 \mathrm{~h}$ dark, $18^{\circ} \mathrm{C}$ photoperiod. Spinach was purchased at a local market.

\section{Construction of GFP reporter plasmids for stable expression in Arabidopsis thaliana}

To express PTOX:GFP fusion in A. thaliana wild type, we PCR-amplified the entire sequence of PTOX using the primers $S a l$ - $N$-ter (CTGGTCGACATGGCGGCGATTTCAGG) and NcoI$C$-ter (TTCCCATGGAACTTGTAATGGATTTCTTGAG) from an A. thaliana cDNA library. The PCR product was cloned into the pBluescript $\mathrm{KS}^{-}$vector (Stratagene). The SalI-NcoI fragment was inserted into the SalI-NcoI digested GFP reporter plasmid Pro35s:SGFP(S65T) to create the Pro35s:PTOX-sGFP(S65T) plasmid. From this construct, we extracted the entire 
cassette Pro $35 s-P T O X-G F P-N o s$ Ter using EcoRI and a partial HindIII digestion. This fragment was purified and inserted into the EcoRI-HindIII digested pEL103 binary vector (kanamycin resistance to transform wild-type plants). Correct orientation and sequence of the inserted fragments were controlled. Plasmids used for Agrobacterium tumefaciens transformation were prepared using the "NucleoSpin Plasmid Kit" (Macherey-Nagel; Germany). The protein level of the GFP:PTOX protein was about three times higher compared to the intrinsic PTOX protein (Supplementary material Fig. S1).

\section{Preparation of crude membrane extract and thylakoid membranes}

Crude extracts: Arabidopsis leaves were infiltrated with uncouplers by placing the petioles into water solutions containing nigericin $(1 \mu \mathrm{M})$, valinomycin $(1 \mu \mathrm{M})$ or just water (control) for $4 \mathrm{~h}$ under low light $\left(8 \mu \mathrm{mol}\right.$ photons $\left.\mathrm{m}^{-2} \mathrm{~s}^{-1}\right)$. The ethanol concentration was $0.01 \%$ in all solutions. Leaves were then exposed for $30 \mathrm{~min}$ to high light $\left(500 \mu \mathrm{mol}\right.$ photons $\left.\mathrm{m}^{-2} \mathrm{~s}^{-1}\right)$. After short homogenization (10 s) in buffer $(0.33 \mathrm{M}$ sorbitol, $60 \mathrm{mM} \mathrm{KCl,} 10 \mathrm{mM}$ EDTA, $1 \mathrm{mM} \mathrm{MgCl}$, $25 \mathrm{mM}$ HEPES pH 7.6 and protease inhibitor cocktail (Sigma-Aldrich, St-Louis, Missouri, United States)) and filtration through 4 layers of mull, the filtrate was separated by centrifugation $\left(5 \mathrm{~min} \times 3000 \mathrm{~g}\right.$ at $4^{\circ} \mathrm{C}$ ) in a supernatant and a membrane fraction. The pellet was resuspended in small amounts of buffer and the chlorophyll concentration determined. Proteins in the supernatant were precipitated with trichloroacetic acid (TCA). $25 \%$ TCA (v/v) was added to the supernatants, 10 min incubated on ice, centrifuged $(15000 \mathrm{~g})$ and the pellet washed 3 times with ice-cold acetone. Finally the pellet was dried and resuspended in $0.1 \mathrm{M}$ TRIS pH 8.0, 4\% SDS before diluting it with sample buffer for SDS-PAGE.

Thylakoid membranes: Spinach leaves were homogenized in a blender for $10 \mathrm{~s}$ using a buffer containing $0.33 \mathrm{M}$ sorbitol, $60 \mathrm{mM} \mathrm{KCl}, 10 \mathrm{mM}$ EDTA, $1 \mathrm{mM} \mathrm{MgCl} 2,25 \mathrm{mM}$ MES, pH 6.1. The slurry was filtered through 4 layers of cheesecloth, and the filtrate was centrifuged $3000 \mathrm{x}$ $\mathrm{g}$ for $3 \mathrm{~min}$ at $4^{\circ} \mathrm{C}$. The supernatant was discarded, and the pellet was resuspended in $0.33 \mathrm{M}$ sorbitol, $60 \mathrm{mM} \mathrm{KCl}, 10 \mathrm{mM}$ EDTA, $1 \mathrm{mM} \mathrm{MgCl} 2,25 \mathrm{mM}$ HEPES, pH 6.7. The suspension was centrifuged $3000 \mathrm{~g}$ for $3 \mathrm{~min}$ at $4^{\circ} \mathrm{C}$. This step was repeated once with $20 \mathrm{mM} \mathrm{KH}_{2} \mathrm{PO}_{4}$, $5 \mathrm{mM} \mathrm{MgCl}_{2}$ as resuspending buffer. Finally, the pellet was resuspended in $0.3 \mathrm{M}$ sucrose, $20 \mathrm{mM} \mathrm{KH}_{2} \mathrm{PO}_{4}, \mathrm{pH}$ 7.6. Before the measurements, thylakoid membranes were incubated for 20 $\mathrm{s}$ in the same buffer containing various concentrations of $\mathrm{MgCl}_{2}$ or $\mathrm{KCl}$.

\section{Expression and purification of MBP-PTOX}


PTOX from Oryza sativa fused with the maltose-binging protein (MBP-OsPTOX) was expressed and purified according to $\mathrm{Yu}$ et al. (2014). MBP protein was expressed and purified in the same way using the empty plasmid.

\section{Liposome preparation}

The thylakoid lipids monogalactosyldiacylglycerol (MGDG), digalactosyldiacylglycerol (DGDG), sulfoquinovosyldiacylglycerol (SQDG), phosphatidylglycerol (PG) and phosphatidylcholine (PC), were purchased from Larodan Fine Chemicals (Malmö, Sweden) and dissolved in chloroform. Lipids were mixed in the following relative molar ratios: $41.2 \%$ MGDG, 26.7\% DGDG, 15.6\% SQDG, 11.5\% PG, and 5\% PC (Natali et al., 2016). $140 \mu 1$ lipids were mixed with $5.84 \mathrm{ml}$ of chloroform. $500 \mu \mathrm{l}$ of the solution was pipetted into a glass vial and the chloroform was slowly removed under $\mathrm{N}_{2}$ vapor in order to form a thin film of lipids on the inner wall of the vial. Lipids were rehydrated (using $500 \mu \mathrm{l}$ buffer (50 mM TRIS-HCl $\mathrm{pH} 8.0,2 \mathrm{mM} \mathrm{KCl}$ or $20 \mathrm{mM} \mathrm{KCl} ; 50 \mathrm{mM}$ MES pH 6.5, $2 \mathrm{mM} \mathrm{KCl}$ or $20 \mathrm{mM} \mathrm{KCl}$ ) and vortexed (three times, each $5 \mathrm{~min}$ ). Extrusion was performed 3 times through a $0.2 \mu \mathrm{m}$ filter using the Extrusion was performed 3 times through a $0.2 \mu \mathrm{m}$ nuclepore membranes (Whatman, GE Healthcare) using a homemade extrusion setup leading to liposomes of about $100 \mathrm{~nm}$ as measured by dynamic light scattering (Nano-S, Malvern, Sysmex, Norderstedt, Germany). 20 $\mu 1$ MBP-OsPTOX ( $1 \mathrm{mg}$ protein $\mathrm{ml}^{-1}$ ) was added to $200 \mu 1$ liposomes, vortexed, deposited on a sucrose gradient $(30 \% / 15 \%)$ and centrifuged at $30000 \mathrm{~g}, 1 \mathrm{~h}, 4^{\circ} \mathrm{C} .200 \mu 1$ were collected from the top of the gradient and used for further analyses.

\section{SDS-Page and Immunoblots}

PTOX and MBP binding to liposomes were resolved by SDS-PAGE (12\%) and proteins were detected using Coomassie Brilliant Blue G250. Protein quantification was done using the Bradford reagent in case of chlorophyll free samples. Protein quantification in chlorophyll containing samples was done using Amidoblack.

Proteins were separated by SDS-PAGE (12\% acrylamide) and immunoblotting using a nitrocellulose membrane. Labelling of the membranes with anti-PTOX antibody raised against the Arabidopsis protein (Josse et al., 2000) and anti-RuBisCo large subunit (RBCL) antibody Agrisera, Vännäs, Sweden) was carried out at room temperature in $50 \mathrm{mM}$ Tris- $\mathrm{HCl} \mathrm{pH} 7.6$, $150 \mathrm{mM} \mathrm{NaCl}, 0.1 \%$ Tween-20 and 5\% non-fat milk powder. After washing, bound antibodies were revealed with a peroxidase-linked secondary anti-rabbit antibody (Agrisera, Vännäs, Sweden) and visualized by enhanced chemiluminescence. 


\section{Chlorophyll fluorescence}

Room temperature chlorophyll fluorescence was measured using a pulse-amplitude modulation fluorometer (DUAL-PAM, Walz, Effeltrich, Germany). The intensity of the measuring light

190

191

192

193

194

195

196

197

198

199

200

201

202

203

204

205

206

207

208

209

210

211

212

213

214

215

216

217

218

219 was sufficiently low so that no increase in the fluorescence $\left(\mathrm{F}_{0}\right)$ was observed. Prior to the measurement, the samples, containing $20 \mu \mathrm{g} \mathrm{chl} \mathrm{ml}^{-1}$ and $1.5 \mu \mathrm{g} \mathrm{ml}^{-1}$ MBP-OsPTOX were darkadapted for $1 \mathrm{~min}$. Fluorescence induction was measured using a multiple turnover flash (duration $300 \mathrm{~ms}$ ).

\section{Confocal laser scanning microscopy}

4 weeks old $A$. thaliana plants expressing GFP-PTOX were used for confocal fluorescence microscopy. Plants were either incubated in red light $\left(150 \mu \mathrm{mol}\right.$ quanta $\left.\mathrm{m}^{-2} \mathrm{~s}^{-1}\right)$ for $30 \mathrm{~min}$ or dark-adapted before mounting leaves for microscopy. In case of measurements using uncoupler, images of the non-treated leaves were first registered and then $1 \mu \mathrm{M}$ nigericin was soaked under the cover slip. Alternatively, the petioles were placed in a $1 \mu \mathrm{M}$ nigericin solution and nigericin was taken up by the transpiration flow during $2 \mathrm{~h}$. 8-bit Images of biological samples and 100 $\mathrm{nm}$ yellow-green and orange fluorescent beads (Fluospheres Carboxylate-modified microspheres, Thermofisher) were collected using a Leica $63 \mathrm{x}$ oil immersion objective (HCX Plan APO CS, NA 1.4, working distance $0.14 \mathrm{~mm}$ ) with an inverted Leica laser-scanning confocal microscope TCS SP5 II (Leica Microsystems, Heidelberg, Germany) equipped with a GaAsP hybrid detection system at Nyquist sampling rate, with the detection pinhole aperture adjusted to 0.6 Airy units. Scanning was performed at $400 \mathrm{~Hz}$. GFP and chlorophyll fluorescence was detected using laser lines $488 \mathrm{~nm}$ and $633 \mathrm{~nm}$, respectively. Detections ranges were set for GFP (495 nm-560 nm) and chlorophyll (644 nm-718 nm). For excitation of GFP fluorescence laser power between 0.15 and $0.3 \mathrm{~mW}$ were chosen (Fig. 4, light: $0.15 \mathrm{~mW}$; dark: $0.28 \mathrm{~mW}$; Fig. 5, $\mathrm{H}_{2} \mathrm{O}$ : $0.23 \mathrm{~mW}$; $\mathrm{H}_{2} \mathrm{O} / \mathrm{Nig}$ : $0.23 \mathrm{~mW}$; Nig: $0.3 \mathrm{~mW}$; Tables 2 and 3: $0.3 \mathrm{~mW}$ ). For chlorophyll fluorescence laser power was adapted to signal strength (Fig. 4: 1.1 mW; Fig. 5, $\mathrm{H}_{2} \mathrm{O}: 0.38 \mathrm{~mW} ; \mathrm{H}_{2} \mathrm{O} / \mathrm{Nig}: 0.38 \mathrm{~mW}$; Nig: $0.18 \mathrm{~mW}$; Tables 2 and 3: $0.46 \mathrm{~mW}$ ). For the detection systems the same gain was chosen for all images (16\% for GaAsP hybrid detector and $711 \mathrm{~V}$ for the photomultiplier tube. Imaging was performed in a temperature-controlled room at $21^{\circ} \mathrm{C}$.

Deconvolution and image treatment: Confocal images of beads and biological data were deconvoluted with the Huygens 3.7 software (Scientific Volume Imaging, Hilversum, Netherlands) using a measured PSF using the Good's (GMLE) algorithm and a signal to noise 
220 ratio of 15 as described in Lam et al. (2017). 3D reconstructions of deconvoluted datasets were 221 carried out with ARIVIS VISION 4D-software. 
223 Previously we reported that PTOX was strongly attached to thylakoid membranes when Arabidopsis leaves had been exposed to high light intensity while it was only weakly attached in leaves incubated in the dark and we hypothesized that PTOX attachment to the membrane depends on the proton motive force (Feilke et al., 2016; Krieger-Liszkay and Feilke, 2016). To show whether the PTOX attachment in the light depends indeed on the proton motive force (pmf), we investigated the effect of uncouplers on the association of PTOX to the thylakoid membrane. We choose the uncouplers valinomycin and nigericin. Unlike valinomycin, a potassium-selective ionophore that dissipates $\Delta \psi$, nigericin abolishes both $\Delta \mathrm{pH}$ and $\Delta \psi$ (Nicholls and Ferguson, 2013). As shown in Fig. 1, less PTOX protein was found in the membrane fractions when leaves had been infiltrated with uncouplers prior to high light exposure. Consistently, more PTOX protein was found in the soluble protein fraction when the leaves had been treated with uncouplers. As loading control RuBisCo was used, that was present in small amounts in the membrane fraction and in large amounts in the fraction of the soluble proteins. No statistically significant difference was found between the two uncouplers used, nigericin and valinomycin. According to the results shown in Fig. 1, the pmf seems to be important for a strong attachment of PTOX to the thylakoid membrane.

To study in vitro the dependency of PTOX association to the thylakoid membrane on $\mathrm{pH}$ value and ion concentration, two approaches were employed using recombinant purified MBP-OsPTOX. In the first approach PTOX affinity to liposomes was investigated, while in the second approach thylakoid membranes were used instead of liposomes. In the first approach PTOX was added to liposomes prepared from a lipid mixture that mimics the lipid composition of the thylakoid membrane. Liposomes were rehydrated in buffers at $\mathrm{pH} 8.0$ or $\mathrm{pH} 6.5$ at two different potassium ion concentrations. $\mathrm{pH} 8.0$ and $\mathrm{pH} 6.5$ were chosen to simulate the $\mathrm{pH}$ values that face the thylakoid membrane in the light: slightly alkaline $\mathrm{pH}$ in the stroma and slightly acidic $\mathrm{pH}$ in the lumen. PTOX attachment to the liposomes was stronger at $\mathrm{pH} 8.0$ than at $\mathrm{pH} 6.5$ (Figure 2), indicating that the membrane association of PTOX is more favorable at slightly alkaline conditions. Furthermore, PTOX attachment at both $\mathrm{pH}$ values was stronger in the presence of $2 \mathrm{mM} \mathrm{KCl}$ than $20 \mathrm{mM} \mathrm{KCl}$. One representative gel from four is shown in Fig. 2. The recombinant PTOX protein used in the present study is a fusion protein between PTOX and the maltose-binding protein MBP. To exclude that the different binding properties of the recombinant protein to liposomes are due to MBP instead of PTOX properties, the same type of experiment was performed with MBP alone (SI Fig. 2). When one compares the protein amounts before and after the sucrose gradient in Fig. 2 and SI Fig. 2, MBP binding was much 
lower than that of MBP-OsPTOX and did neither depend on the ion concentration nor on the $\mathrm{pH}$-value. Therefore we conclude that it was indeed the binding of PTOX that was sensitive to both, the $\mathrm{pH}$ value and the ion concentration. Next, we investigated whether the lipid composition of the liposomes influenced the attachment of PTOX. When the charged lipids PG or SQDG were omitted from the liposomes, no difference in PTOX attachment was observed compared with the results obtained using liposomes prepared with the standard lipid composition (SI Fig. 3).

In the second approach MBP-OsPTOX was added to isolated thylakoid membranes. Binding was strongest in the presence of $1 \mathrm{mM} \mathrm{MgCl} 2$ and decreased at higher $\mathrm{MgCl}_{2}$ concentrations (Fig. 3). Fv/Fm values were similar at $1 \mathrm{mM}$ and at $5 \mathrm{mM} \mathrm{MgCl}$, indicating that the same degree of stacking of the thylakoid membranes (Barber et al., 1980) was obtained with the two $\mathrm{MgCl}_{2}$ concentrations (Table 1). When $\mathrm{KCl}$ was used instead of $\mathrm{MgCl}_{2}$, a weaker binding was observed at $20 \mathrm{mM} \mathrm{KCl}$ than at $2 \mathrm{mM} \mathrm{KCl}$ or in the absence of added $\mathrm{KCl}$. Binding of MBP-OsPTOX to the thylakoid membrane without addition of salts may be either due to the ion concentration in the thylakoid membranes remaining from the preparation or to residual binding independent of the ionic strength of the medium. PTOX activity was monitored by chlorophyll fluorescence induction (SI Fig. 4). Addition of MBP-OsPTOX affected fluorescence induction at $\mathrm{pH} 8.0$ but not at $\mathrm{pH} 6.5$.

We used GFP-labelled AtPTOX expressed in Arabidopsis thaliana and confocal fluorescence microscopy to investigate changes in PTOX localization between light-adapted and dark-adapted chloroplasts. Expression of GFP-PTOX led to a faster quenching of variable chlorophyll fluorescence upon onset of actinic light but had little effect on photochemical quenching and non-photochemical quenching when measured on non-stressed plants taken from the growth cabinet (SI Fig. 5). Localization of the GFP fluorescence changed in the chloroplasts when chloroplasts from high light-treated and dark-adapted plants were compared as shown in Fig. 4 for chloroplasts of guard cells. In light-adapted plants GFP fluorescence was homogenously distributed over the whole chloroplasts while in dark-adapted plants GFP fluorescence was visible as characteristic spots restricted to small areas at the outer edges of the chloroplasts. Infiltrating leaves of high light exposed plants with nigericin led also to a more spotted distribution of the GFP fluorescence than observed in the absence of the uncoupler as shown in Fig. 5. In chloroplasts of wild-type plants no green autofluorescence spots were seen using the same setting of the microscope (SI Fig. 6). The analysis of more than hundred chloroplasts shows a large increase in chloroplasts with spots of GFP fluorescence in the presence of nigericin compared to a more homogenous distribution in the absence of the 
uncoupler (Table 2). Next we analyzed the size of the GFP fluorescence spots (Table 3). The majority of the volume of the spots was smaller than $100 \mu \mathrm{m}^{3}$, however, also much larger spots were found. Spot size distribution is heterogeneous with average volume ranges from $0.034 \pm 0.008$ to $0.687 \pm 0.159 \mu \mathrm{m}^{3}$.

\section{Discussion}

\section{Effect of pH and ionic strength on the attachment of PTOX to the membrane}

Using leaves, thylakoid membranes and liposomes, we demonstrated here that the association of PTOX to the membrane was affected by uncouplers (Fig. 1, 5), by $\mathrm{pH}$ and by ion concentration (Figs 2, 3). According to our hypothesis, association of PTOX with the membrane is required to allow the access to its lipophilic substrate plastoquinol. A temporary, reversible attachment to the membrane is known to regulate the activity of certain enzymes. A change in $\mathrm{pH}$, inducing protonation/deprotonation and in parallel, a change in the protein charge, induces for example membrane binding of hisactophilin, a cytoskeletal linker protein in Dictyostelium (Scheel et al., 1989). Another example of an enzyme known to associate with the thylakoid membrane in a pH-dependent manner is the violaxanthin de-epoxidase (Hager and Holocher, 1994; Hieber et al., 2002). The $\mathrm{pH}$ value of the stroma is known to increase upon illumination (Heldt et al., 1973), and the light-induced proton increase in the lumen is electrically counterbalanced by ion fluxes through $\mathrm{Cl}^{-}$- (Schönknecht et al., 1988) and $\mathrm{K}^{+}$-channels (Tester and Blatt, 1989; Carraretto et al., 2013). We suppose that both, the $\mathrm{pH}$ value and the ion concentration, are crucial for the attachment of PTOX with the membrane since both uncouplers nigericin and valinomycin led to a dissociation of a fraction of PTOX from thylakoid membranes in infiltrated leaves (Fig 1). According to reconstitution experiments using liposomes or isolated thylakoid membranes and recombinant Os-PTOX fused with the maltose binding protein, a more alkaline $\mathrm{pH}$ and a $\mathrm{KCl}$ or $\mathrm{MgCl}_{2}$ concentration in the lower millimolar range favoured the attachment of PTOX to the membrane (Figs 2, 3). We propose that lowering the $\mathrm{pH}$ from 8.0 to 6.5 induces either a conformational change in the PTOX protein structure that leads to a dissociation of the protein from the membrane or leads to protonation of amino acid residues that provide positive charges that are crucial for the membrane association. Since PTOX attachment is stronger at $\mathrm{pH} 8.0$ than at $\mathrm{pH} 6.5$ we suppose that PTOX is active at the stroma facing side of the membrane. 
PTOX like AOX, the alternative oxidase of the mitochondria, is composed of a fourhelix bundle that harbors the chelating sites for the diiron center and of two additional amphipathic helices that dock to one of the leaflets of the membrane (Nawrocki et al., 2015). Compared to the sequence of PTOX2 from Chlamydomonas reinhardtii that was used for modelling the membrane interaction by Nawrocki and co-workers, the length of the amphipathic helices is shorter in PTOX from higher plants and more similar in length to AOX. For AOX from Trypanosoma brucei a crystal structure is available (Shiba et al., 2013). The interaction of the AOX dimer with the membrane was modelled and, according to this model, five arginine residues are distributed along a boundary between the hydrophobic and hydrophilic regions of the dimer surface and the membrane. Several arginine residues are present in the predicted membrane association domains of the PTOX and may be important for the membrane association of the protein. They may interact with the negatively charged phospholipid head groups of the membrane. The arginine residues should remain charged at both $\mathrm{pH}$ values used here. Therefore, it seems more likely that a conformational change of the protein is responsible for its dissociation from the membrane at $\mathrm{pH} 6.5$ than a protonation of an amino acid that permits the anchoring of the protein with the membrane. Hydrophobic interactions may be also important for the interaction between the membrane and the PTOX. $\mathrm{pH}$ changes and changes in the ion concentration can affect this kind of interaction. A specific role of either SQDG or PQ on the PTOX interaction with the membrane can be excluded since omission of either of the charged lipids from the lipid mixture for liposome production did not affect PTOX affinity (Fig. SI 2).

\section{Localization of PTOX in the chloroplast}

Confocal microscopy data suggest that there is a pool of soluble PTOX that accumulates in spots in the dark while in the light PTOX is more homogenously distributed in a kind of network among the thylakoid membranes. Further investigations are needed to understand the nature of the PTOX accumulation/dissociation. We propose a dynamic model of PTOX localization inside the chloroplast: If the proton gradient across the membrane reaches a certain threshold value, PTOX moves to the margin region or the grana stacks of the thylakoid membrane where it has access to its substrate and fulfills its function keeping the redox state of the PQ pool poised. Under conditions of a lower proton motive force it detaches from the membrane or localizes in parts of the membrane system where it is inactive in respect to photosynthetic electron transport. In the light, an increase in $\mathrm{pH}$ and in ion concentration in the stroma may dissolve PTOX spots and facilitate the binding of PTOX to the membrane. In addition to soluble 
PTOX, PTOX seems to be located in its resting state in high concentrations at the stroma lamellae or in specific membrane domains seen as spots by confocal microscopy. The diffraction-limited resolution of confocal microscopy does not allow to distinguish between spots in the stroma and spots that are formed at a specific membrane domain. Stepien and Johnson (2018) showed that PTOX overexpressed in Eutrema salsugineum moved from the stroma lamellae to the grana stacks upon exposure to salt stress. Such a movement is consistent with our model, however, accumulation of PTOX in high concentrations at the stroma lamellae are required to explain the spot formation. In contrast to Stepien and Johnson (2018) who proposed that PTOX translocates in an unknown way from the stroma-facing side to the luminal side of the membrane and that it is active in the thylakoid lumen, our data suggest that PTOX is active at the stromal side of the membrane at slightly alkaline $\mathrm{pH}$ values.

The different distribution of PTOX within the chloroplast may be caused by both, differences in the ratio between membrane-bound PTOX and soluble PTOX and by different oligomerization states of PTOX. PTOX may accumulate in high concentrations in its soluble form forming the spots and may dissociate into smaller units upon changes in $\mathrm{pH}$ and binding to the membrane. These spots may be part of a cellular structure that contains not only PTOX in high concentration but also other components and proteins. A large part of the spots falls into the size category of a known specific cellular structure, namely plastoglobuli. Plastoglobuli have a diameter of 45 to $60 \mathrm{~nm}$ (Austin II et al., 2006) in chloroplast of non-stressed plants. However, also smaller and larger PTOX spots were found in the chloroplasts analyzed here. Furthermore, the spot size does not increase upon light stress (SI Fig. 7). This finding does not correlate well with plastoglobuli. Most probably, the GFP spots represent accumulations of PTOX in a structure of variable size different to regular plastoglobuli.

The catalytically functional unit of PTOX consists most likely of a dimer as it is the case for AOX (Shiba et al., 2013). MBP-OsPTOX tends to form different oligomeric states depending on the used detergent. In the presence of n-octyl $\beta$-D-glucopyranoside MBP-PTOX was found mainly as a tetramer (Yu et al., 2014) while it was mainly in a dimeric form in the presence of $\beta$-dodecyl-maltoside (SI Fig. 8). The native protein and the GFP-fusion protein may form larger oligomers than MBP-PTOX, since fusion with MBP increases the solubility of the protein. Different oligomerization states of phytoene desaturase, an enzyme of the carotenoid biosynthesis pathway, have been reported previously. This enzyme does exist in a soluble form and in a membrane-associated form where it has access to its lipophilic substrate. Phytoene desaturase forms higher order oligomeric species with two distinguishable structures consisting either of rings or stacks (Gemmecker et al., 2015). Rings were ascribed to a tetramer which 
assembled into stacked tubular structures of a length between $15-30 \mathrm{~nm}$. The stacks represented the soluble form while the active form attached to liposomes was a single tetrameric ring.

Knowledge about the geometry of the membrane system is required to understand relocalization of a protein like PTOX. Higher plant thylakoid membranes are organized in grana stacks, composed of cylindrical stacked membranes of 350-600 nm in diameter, interconnected by unstacked stroma lamellae, and forming a continuous complex structured membrane network that separates the thylakoid lumen from the stroma. It has been observed that the overall thylakoid architecture is highly dynamic (Kirchhoff, 2019; Pribil et al., 2014). It is likely that changes in the size of the lumen or a partial destacking of grana control transport processes within the thylakoid membrane system and may control also the localization of proteins in the stroma which is densely packed with proteins. In the light, the building up of the proton motive force may cause swelling of the lumen induced by influx of protons into the lumen (Kirchhoff et al., 2011; Ruban and Johnson, 2015) and may affect thereby protein localization in the stroma. The ion concentration in the stroma increases when the proton motive force is high. A change of the ion concentration in the stroma may affect the solubility of proteins, the stability of larger protein complexes and may allow the accumulation of PTOX in spots.

Further studies are required to get insights into the arrangement of PTOX at the membrane, to understand the mechanism behind PTOX accumulation into spots, to investigate whether other proteins or lipids than PTOX are present in the spots and to study whether PTOX activity correlates with its distribution (network-like structure versus spots) within the chloroplast.

\section{Acknowledgements}

A.K.L., E. M., M. J. and M.P. benefit from the support of the LabEx Saclay Plant Sciences-SPS (ANR-10-LABX-0040-SPS) and the French Infrastructure for Integrated Structural Biology (FRISBI) ANR-10-INSB-05. 


\section{References}

Austin II JR, Frost E, Vidi PA, Kessler F Staehelin LA. 2006. Plastoglobules are lipoprotein subcompartments of the chloroplast that are permanently coupled to thylakoid membranes and contain biosynthetic enzymes. Plant Cell 18, 1693-1703. https://doi.org/10.1105/tpc.105.039859

Barber J., Chow W.S., Scoufflaire C., Lannoye R. 1980. The relationship between thylakoid stacking and salt induced chlorophyll fluorescence changes. Biochimica et Biophysica Acta 591, 92-103. https://doi.org/10.1016/0005-2728(80)90223-6

Carol P, Stevenson D, Bisanz C, Breitenbach J, Sandmann G, Mache R, Coupland G, Kuntz M. 1999. Mutations in the Arabidopsis gene IMMUTANS cause a variegated phenotype by inactivating a chloroplast terminal oxidase associated with phytoene desaturation. Plant Cell 11, 57-68. https://doi.org/10.1105/tpc.11.1.57

Carraretto L, Formentin E, Teardo E, Checchetto V, Tomizioli M, Morosinotto T, Giacometti, GM, Finazzi G, Szabó I. 2013. A thylakoid-located two-pore K+ channel controls photosynthetic light utilization in plants. Science 342, 114-118. https://doi.org/10.1126/science.1242113

Feilke K, Streb P, Cornic G, Perreau F, Kruk J, Krieger-Liszkay A. 2016. Effect of Chlamydomonas plastid terminal oxidase 1 expressed in tobacco on photosynthetic electron transfer. Plant Journal 85, 219-228. https://doi.org/10.1111/tpj.13101

Gemmecker S, Schaub P, Koschmieder J, Brausemann A, Drepper F, Rodriguez-Franco M, Ghisla S, Warscheid B, Einsle O, Beyer P. 2015. Phytoene Desaturase from Oryza sativa: Oligomeric Assembly, Membrane Association and Preliminary 3D-Analysis. PLoS One. 10:e0131717. https://doi.org/10.1371/journal.pone.0131717

Hager A, Holocher K. 1994. Localization of the xanthophyll-cycle enzyme violaxanthin deepoxidase within the thylakoid lumen and abolition of its mobility by a (light-dependent) $\mathrm{pH}$ decrease. Planta 192, 581-589. https://doi.org/10.1007/BF00203597

Heldt HW, Werdan K, Milovancev M, Geller G. 1973. Alkalization of the chloroplast stroma caused by light-dependent proton flux into the thylakoid space. Biochimica Biophysica Acta 314, 224-241. https://doi.org/10.1016/0005-2728(73)90137-0

Hieber AD, Bugos RC, Verhoeven AS, Yamamoto HY. 2002. Overexpression of violaxanthin de-epoxidase: properties of C-terminal deletions on activity and $\mathrm{pH}$-dependent lipid binding. Planta 214, 476-483. https://doi.org/10.1007/s00425-001-0704-2

Ivanov, AG, Rosso D, Savitch LV, Stachula P, Rosembert M, Oquist G, Hurry V, Hüner NPA. 2012. Implications of alternative electron sinks in increased resistance of PSII and PSI photochemistry to high light stress in cold-acclimated Arabidopsis thaliana. Photosynthesis Research 113, 191-206. https://doi.org/10.1007/s11120-012-9769-y

Josse EM, Simkin AJ, Gaffé J, Labouré AM, Kuntz M, Carol P. 2000. A plastid terminal oxidase associated with carotenoid desaturation during chromoplast differentiation. Plant Physiol 123, 1427-1436. https://doi.org/10.1104/pp.123.4.1427

Ruban AV, Johnson MP. 2015. Visualizing the dynamic structure of the plant photosynthetic membrane. Nature plants 1,15161. https://doi.org/10.1038/nplants.2015.161

Kirchhoff H, Hall C, Wood M, Herbstová M, Tsabari O, Nevo R, Charuvi D, Shimoni E, Reich Z. 2011. Dynamic control of protein diffusion within the granal thylakoid lumen. Proceedings of the National Academy of Sciences U S A 108, 20248-20253. https://doi.org/10.1073/pnas.1104141109

Kirchhoff H. 2019. Chloroplast ultrastructure in plants. New Phytologist 223, 565-574. https://doi.org/10.1111/nph.15730 
Krieger-Liszkay A, Feilke K. 2016. The dual role of the Plastid Terminal Oxidase PTOX: Between a protective and a pro-oxidant function. Frontiers in Plant Science 6,1147. https://doi.org/10.3389/fpls.2015.01147

Lam F, Cladière D, Guillaume C, Wassmann K, Bolte S. 2017. Super-resolution for everybody: An image processing workflow to obtain high-resolution images with a standard confocal microscope. Methods 115, 17-2. https://doi.org/10.1016/j.ymeth.2016.11.003

Laureau C, De Paepe R, Latouche G., Moreno-Chacón M, Finazzi G, Kuntz M, Cornic G, Streb P. 2013. Plastid terminal oxidase (PTOX) has the potential to act as a safety valve for excess excitation energy in the alpine plant species Ranunculus glacialis L. Plant Cell \& Environment 36, 1296-1310. https://doi.org/10.1111/pce.12059

Lennon AM, Prommeenate P, Nixon PJ. 2003. Location, expression and orientation of the putative chlororespiratory enzymes, ndh and immutans, in higher-plant plastids. Planta 218, 254-260. https://doi.org/10.1007/s00425-003-1111-7

Natali A, Gruber JM, Dietzel L, Stuart MCA, van Grondelle R, Croce R. 2016. Lightharvesting complexes (LHC) cluster spontaneously in membrane environment leading to shortening of their excited state lifetimes. Journal of Biological Chemistry 291, 1673016739. https://doi.org/10.1074/jbc.M116.730101

Nawrocki WJ, Tourasse NJ, Taly A, Rappaport F, Wollman FA. 2015. The plastid terminal oxidase: its elusive function points to multiple contributions to plastid physiology. Annual Reviews of Plant Biology 66, 49-74. https://doi.org/10.1146/annurev-arplant-043014114744

Nicholls DG, Ferguson SJ. 2013. Bioenergetics. $4^{\text {th }}$ edition. London: Academic Press, London UK, p. 19

Ollion J, Cochennec J, Loll F, Escudé C, Boudier T. 2013. TANGO: A Generic Tool for High-throughput 3D Image Analysis for Studying Nuclear Organization. Bioinformatics 29, 1840-1841. https://doi.org/10.1093/bioinformatics/btt276

Pribil M, Labs M, Leister D. 2014. Structure and dynamics of thylakoids in land plants. Journal of Experimental Botany 65, 1955-1972. https://doi.org/10.1093/jxb/eru090

Quiles MJ. 2006. Stimulation of chlororespiration by heat and high light intensity in oat plants. Plant Cell \& Environment 29, 1463-1470. https://doi.org/10.1111/j.13653040.2006.01510.x

Schönknecht G, Hedrich R, Junge W, Raschke K. 1988. A voltage-dependent chloride channel in the photosynthetic membrane of a higher plant. Nature 336, 589-592. https://doi.org/10.1038/336589a0

Shiba T, Kido Y, Sakamoto K, Inaoka DK, Tsuge C, Tatsumi R, Takahashi G, Balogun EO, Nara T, Aoki T, Honma T, Tanaka A, Inoue M, Matsuoka S, Saimoto H, Moore AL, Harada S, Kita K. 2013. Structure of the trypanosome cyanide-insensitive alternative oxidase. Proceedings of the National Acaddemy of Sciences U S A 110, 4580-4585. https://doi.org/10.1073/pnas.1218386110

Stepien P, Johnson GN. 2009. Contrasting responses of photosynthesis to salt stress in the glycophyte Arabidopsis and the halophyte Thellungiella: role of the plastid terminal oxidase as an alternative electron sink. Plant Physiology 149, 1154-1165. https://doi.org/10.1104/pp.108.132407

Tester M, Blatt MR. 1989. Direct measurement of K channels in thylakoid membranes by incorporation of vesicles into planar lipid bilayers. Plant Physiology 91, 249-252.

Trouillard M, Shahbazi M, Moyet L, Rappaport F, Joliot P.A., Kuntz M., Finazzi G. 2012. Kinetic properties and physiological role of the plastoquinone terminal oxidase (PTOX) in a vascular plant. Biochiimica et Biophysica Acta 1817, 2140-2148. https://doi.org/10.1104/pp.91.1.249 
Scheel J, Ziegelbauer K, Kupke T, Humbel BM, Noegel AA, Gerisch G, Schleicher M. 1989. Hisactophilin, a histidine-rich actin-binding protein from Dictyostelium discoideum. Journal of Biological Chemistry 264, 2832-2839.

Wetzel CM, Jiang CZ, Meehan LJ, Voytas DF, Rodermel SR. 1994. Nuclear-organelle interactions: the immutans variegation mutant of Arabidopsis is plastid autonomous and impaired in carotenoid biosynthesis. Plant Journal 6, 161-175. https://doi.org/10.1046/j.1365-313X.1994.6020161.x

Yu Q, Feilke K, Krieger-Liszkay A, Beyer P. 2014. Functional and molecular characterization of plastid terminal oxidase from rice (Oryza sativa). Biochimica et Biophysica Acta 1837, 1284-1292. https://doi.org/10.1016/j.bbabio.2014.04.007 


\begin{tabular}{|c|c|}
\hline salt concentration & $\mathrm{Fv} / \mathrm{Fm}$ \\
\hline no salt added & $0.79 \pm 0.15$ \\
\hline $1 \mathrm{mM} \mathrm{MgCl}_{2}$ & $0.86 \pm 0.05$ \\
\hline $5 \mathrm{mM} \mathrm{MgCl}_{2}$ & $0.86 \pm 0.07$ \\
\hline $2 \mathrm{mM} \mathrm{KCl}$ & $0.82 \pm 0.10$ \\
\hline $20 \mathrm{mM} \mathrm{Kcl}$ & $0.82 \pm 0.10$ \\
\hline
\end{tabular}

536

537 Isolated thylakoid membranes were diluted to $20 \mu \mathrm{g} \mathrm{chl} \mathrm{ml}^{-1}$ in 0.3 sorbitol, $25 \mathrm{mM}$ HEPES $538 \mathrm{pH} 7.6$ containing the indicated concentration of $\mathrm{MgCl}_{2}$ or $\mathrm{KCl}$ and chlorophyll fluorescence 539 induction curves were measured using a saturating light pulse after 5 min dark adaptation.

540

541

542

543 
Table 2: Distribution of GFP-PTOX in leaves in water and in nigericin

545

\begin{tabular}{|l|c|c|}
\hline & water & nigericin \\
\hline Number chloroplasts with spots & 12 & 93 \\
\hline Number of chloroplasts counted & 145 & 120 \\
\hline Images analyzed & 14 & 13 \\
\hline
\end{tabular}

546 Leaves were illuminated for $15 \mathrm{~min}$ on the microscope stage using the continuous light source 547 of the microscope and additionally an LED lamp emitting at $650 \mathrm{~nm}\left(160 \mu \mathrm{mol}\right.$ quanta $\left.\mathrm{m}^{-2} \mathrm{~s}^{-1}\right)$ 548 before taking images. Nigericin was added by placing a drop in close proximity to the cover 549 slip and letting it enter into the leaf by diffusion.

550 


\begin{tabular}{|c|c|c|c|}
\hline Spot groups & $\begin{array}{c}\text { volume }\left(\mu \mathrm{m}^{3}\right) \\
\text { of spot groups }\end{array}$ & $\begin{array}{l}\text { Average volume }\left(\mu \mathrm{m}^{3}\right) \\
\text { per group }\end{array}$ & number of spots \\
\hline 1 & $0.023-0.049$ & $0.034 \pm 0.008$ & 56 \\
\hline 2 & $0.050-0.099$ & $0.068 \pm 0.012$ & 62 \\
\hline 3 & $0.100-0.149$ & $0.124 \pm 0.014$ & 25 \\
\hline 4 & $0.149-0.290$ & $0.203 \pm 0.042$ & 28 \\
\hline 5 & $0.300-0.490$ & $0.357 \pm 0.038$ & 18 \\
\hline 6 & $0.500-1.000$ & $0.687 \pm 0.159$ & 7 \\
\hline
\end{tabular}

554 Confocal image stacks of dark-adapted leaves were processed with ImageJ (3D-iterative 555 thresholding plugin (Ollion et al., 2013) to calculate spot volumes of PTOX-GFP labeled 556 structures.

557

558

559

560 


\section{Figure 1. PTOX attachment depends on the proton motive force.}

563

Arabidopsis leaves were exposed for $30 \mathrm{~min}$ to high light $\left(500 \mu \mathrm{mol}\right.$ photons $\left.\mathrm{m}^{-2} \mathrm{~s}^{-1}\right)$ in the absence or presence of $1 \mu \mathrm{M}$ uncouplers. Leaf extracts were separated by centrifugation into a membrane fraction and a fraction containing soluble proteins. PTOX content of the membrane fraction was analyzed by SDS-PAGE and immunoblotting. Gels were loaded based on chlorophyll content (10 $\mu \mathrm{g}$ Chl per lane). Upper panels: Representative blots; C: no addition; N: Nigericin; V: Valinomycin. Two different exposure times were used for the blot showing the supernatants to allow discrimination between the two bands. Lower panels densityiesof the PTOX band in the membrane fractions (left) and the soluble protein fraction (right) from uncoupler-treated leaves were normalized to the density of the band of control (mean $\pm \mathrm{SE}, 3$ blots, each with proteins from different preparations, were used for the statistical analysis. The significance level obtained by Student's t-test $(P<0.05)$ is indicated by letters.

Figure 2. PTOX attachment to liposomes as a function of $\mathrm{pH}$ and salt concentration. Purified recombinant MBP-OsPTOX protein (1 $\mu \mathrm{g}$ protein) was adsorbed to liposomes. Not absorbed PTOX was removed by centrifugation using sucrose gradients and the PTOX content of the recovered liposomes was analyzed by SDS-Page and CBB staining. PTOX attachment is shown at $\mathrm{pH} 6.5$ and $\mathrm{pH} 8.0$ in the presence of 2 and $20 \mathrm{mM} \mathrm{KCl}$, respectively.

Figure 3. PTOX attachment to thylakoid membranes as a function of the $\mathrm{MgCl}_{2}$ or $\mathrm{KCl}$ concentration.

Purified recombinant MBP-OsPTOX protein $\left(1.5 \mu \mathrm{g} \mathrm{ml}^{-1}\right)$ was adsorbed to spinach thylakoid membranes $\left(10 \mu \mathrm{g} \mathrm{Chl} \mathrm{ml}^{-1}\right)$ at $\mathrm{pH} 7.6$ at the indicated ion concentrations. The membranes were pelleted before deposition of the samples on the gel (SDS-PAGE). PTOX amounts were revealed by immunoblotting.

\section{Figure 4}

Confocal fluorescence microscopy of guard cells of A. thaliana expressing GFP-PTOX Confocal images of chloroplasts expressing GFP of plants exposed to light (light, upper panels) throughout the experiment and plants kept in the dark before imaging (dark, lower panels). Upper panels represent single fluorescence images (A) and 3-dimensional views of image stacks of control plants (A', A'). Lower panels represent single fluorescence images (B) and 3-dimensional views of image stacks of plants kept in the dark (B', B'). 
595 Fluorescent images (A, B) represent chlorophyll fluorescence (red) and GFP fluorescence 596 (green) of stomata, with arrowheads marking the position of two chloroplasts shown in higher 597 magnification below ( $a, a^{\prime}, b$ and b') and on the respective 3D-images. Scale bars are $5 \mu \mathrm{m}$.

598 Note the distribution of GFP in control plants (network) and dark treated plants (spots). Young 599 leaves (3 week old plants) were chosen for the images.

600

\section{$601 \quad$ Figure 5}

602 Effect of the uncoupler nigericin on GFP-PTOX localisation in guard cells.

603 Leaves were exposed to red light $\left(150 \mu \mathrm{mol}\right.$ quanta $\left.\mathrm{m}^{-2} \mathrm{~s}^{-1}\right)$ for $30 \mathrm{~min} .1 \mu \mathrm{M}$ nigericin was 604 soaked under the cover slip for the infiltrated leaves. Alternatively, the petioles were placed in 605 a $1 \mu \mathrm{M}$ nigericin solution and nigericin was taken up by the transpiration flow during $2 \mathrm{~h}$ 606 (incubated leaves). Scale bars are $5 \mu \mathrm{m}$.

607

608

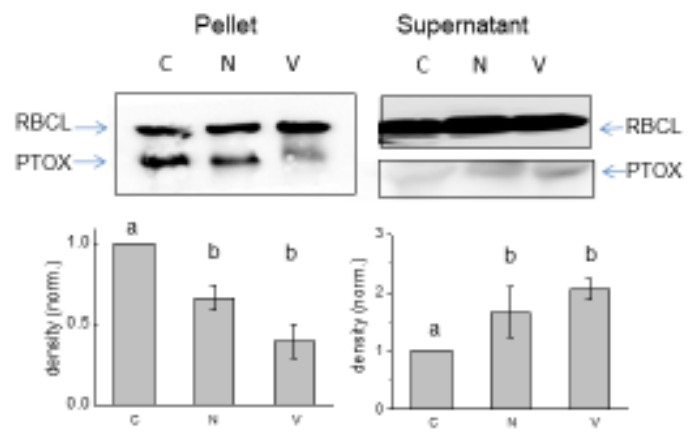

Figure 1. PTOX attachment depends on the proton motive forse. 


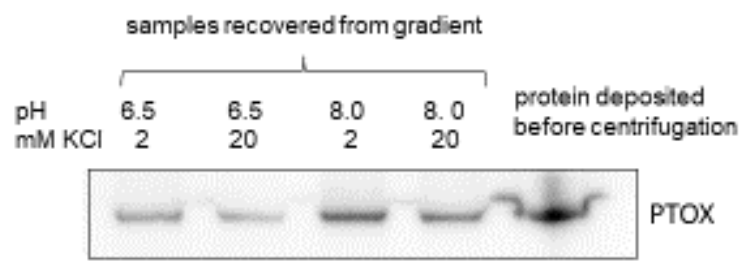

Figure 2. PTOX attachment to liposomes as a function of pH and lonic strength

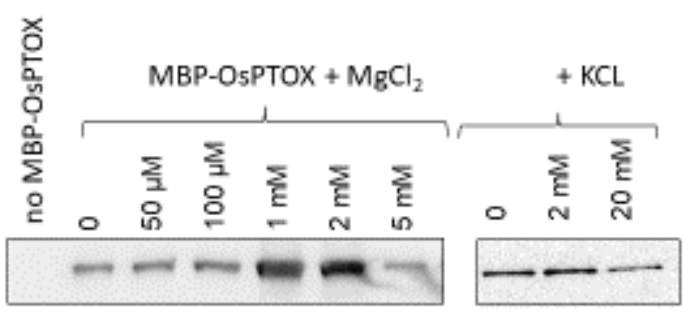

Figure 3. PTOX attachment to thylakoid membranes as a function of the $\mathrm{MgCl}_{\mathrm{Z}}$ or WCl eoncentration.

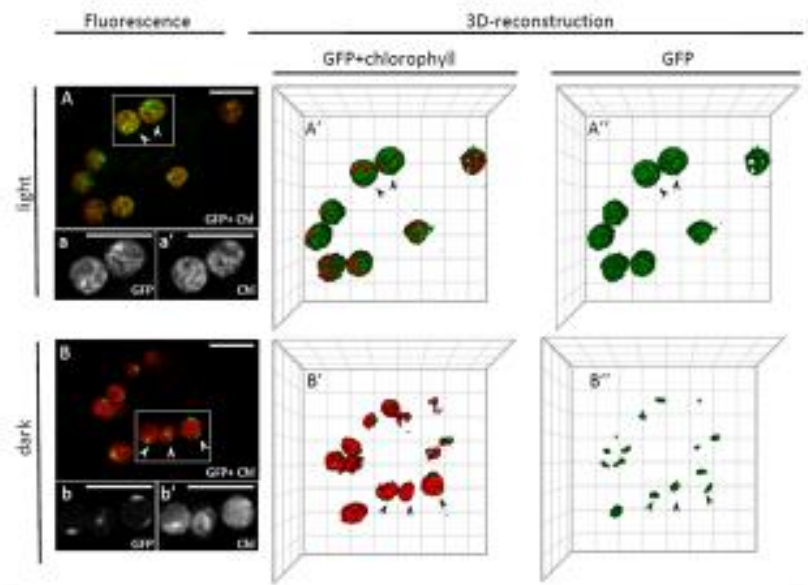

Figure 4. Confocal fluerestente mieroscopy of guard cells of A. thatiana expressing GP-PTOX 


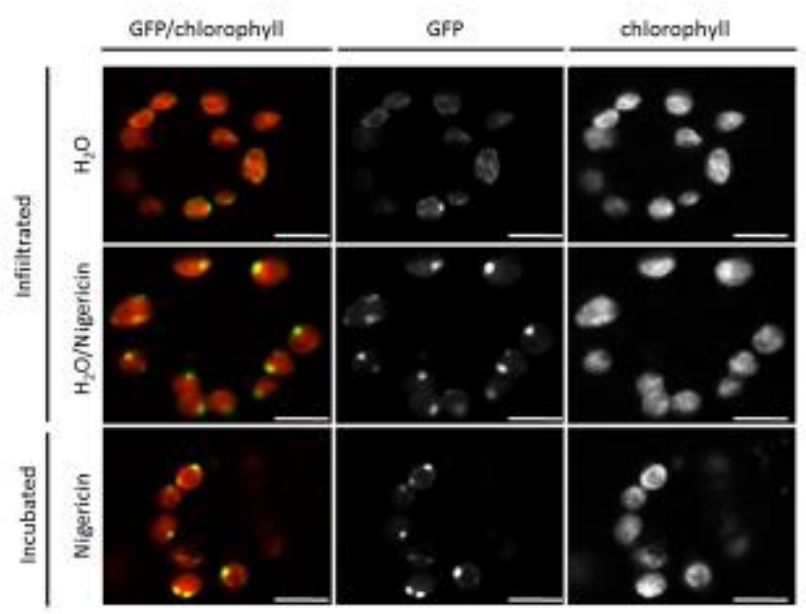

Figure S. Effect of the unsoupler nigericin on GFp-prox localication in guard cells 